Coastal Dynamics 2009

Paper No.

\title{
POLLUTANT DISPERSION AROUND THE PERIMETER OF A COASTAL HEADLAND
}

\author{
Fu Ee Tang ${ }^{1}$
}

\begin{abstract}
The dispersion of point and nonpoint source pollutants may be complicated by the existence of coastal geographical features such as headlands. When coastal flow passes a headland on the coastal line, a recirculation zone will be formed as the flow separates at the headland toe. The experimental investigation was carried out in a purpose-built flume, located at the Manchester Tidal flow Facility (MTF). From the experiments, if pollutant is discharged at different locations around the perimeter of a headland, the potential of pollutant trapping around the headland and its generated recirculation zone will change accordingly. This study investigates the characteristics of pollutant dispersion in the region around the headland perimeter. Effective pollutant dispersion at the recirculation zone will be crucial to maintain the water quality of the region.
\end{abstract}

Key words: Coastal headland, recirculation zone, pollutant trapping, pollutant dispersion

\section{Introduction}

Coastal geographical features such as headlands, promontories and islands may trap water and complicate the dispersion of pollutants discharged in these shallow coastal areas. If pollutant is discharged at different locations around the perimeter of a headland, the potential of pollutant trapping around the headland will change accordingly. Less trapping can be expected if the discharge point is located toward the toe of the headland which is further into the ocean. Since the water depths in coastal areas are typically much smaller than the horizontal scale (here the size of the headland), it is thus known as the shallow water layer. Therefore, the study of coastal headland wakes ought to be put into the context of the shallow water flows. The shallow water layer has been studied extensively in previous research work. Some relevant examples of these studies are as follows. Wolanski, et al (1984), and Ingram and Chu (1987) have shed more light on headland and island flow behaviour. In Ingram and Chu (1987) the bed friction effect on the transverse shear was investigated. Chen and Jirka (1991, 1995 and 1997) have studied pollutant mixing, flow patterns and hydrodynamic instabilities in wake flows behind an island in shallow waters.

When coastal flow passes a headland on the coastal line, a recirculation zone will normally be formed as the flow separates at the headland toe, which is similar to the sudden open channel flow expansion. The steady flow cases for this situation have been studied previously. For example, Babarutsi, et al. (1989) and Babarutsi and Chu (1991) have studied the characteristics of a sudden open channel flow expansion. MacDonald and Jirka (1997) have studied flow behaviour of headland wakes in shallow flow, using several bluff bodies to represent their headland.

The authors have studied coastal headland wakes experimentally using the Manchester Tidal Facility (MTF) and the UK Coastal Research Facility (UKCRF) for both steady and unsteady flows. Lloyd et al (2001) studied shallow water flow around an island in MTF. The Particle Tracking Velocimetry (PTV) technique was used and compared with numerical simulation. For flows behind a headland, Chen et al (2004) has put both UKCRF and MTF data together and a nice classification of wake formation of a headland has been achieved in oscillatory flow according to the Keulegan-Carpenter (KC) number. Some work has been done earlier by the authors (Chen, et al 2003) as well for the same coastal headland.

In real-life situations sometimes near-stagnant flow or very slow-moving flow may occur due to certain coastal bathymetries. The near-stagnant flow can give rise to hazardous situations that harm the wildlife

${ }^{1}$ Civil and Construction Eng. Dept., Curtin University of Technology Sarawak Campus, CDT 250, 98009 Miri, Sarawak, Malaysia. tang.fu.ee@curtin.edu.my 
and inhabitants of these waters and areas. In the region around a headland these flow situations may occur in a secluded bay or cove area, and flow velocities near the shore will certainly be very low. So far, pollutant dispersion around a headland remains poorly understood, especially the effect of varying the different locations for discharging around the perimeter of the headland.

A fundamental study is carried out experimentally here to study pollutant dispersion in steady flow at the region around the perimeter of a coastal headland. This study aims to investigate the characteristics of flow dispersion and trapping behaviour by simulating pollutant discharge around the perimeter of a simplified headland model in a large-scale flume. The MTF is capable of generating flows with Reynolds numbers of sufficiently large magnitude. The release of pollutants is simulated by fluorescent dye. Quantitative measurements of dye concentrations were made with a PLIF system and data collaboration procedures which have been developed in the authors previous studies (Chen and Jirka, 1999; Chen et al 2004). The raw experiment images are calibrated and processed, to produce dye decay profiles that characterize dye decay behaviour in these areas.

\section{Experimental Methodology}

Due to space constraints, only an overview of the experimental set-up will be presented here. The experimental set-up had been presented in Chen (2005). The experiments were conducted in a purposebuilt shallow water flume, with full dimensions $11 \mathrm{~m} \times 3.3 \mathrm{~m} \times 0.2 \mathrm{~m}$. A 1:20 plane beach was constructed on one side as shown in Figure 1. The sloped beach extended laterally from the bed of the flume to near the top of the sides. The bed of the flume was covered in $5 \mathrm{~mm}$ median diameter gravel, the equivalent roughness, $\mathrm{k}_{\mathrm{s}}$ was taken as $2 \mathrm{D}_{\mathrm{g}}$. A uni-directional variable speed pump and an arrangement of four valves was used. The headland model consisted of a conical, ellipsoid, head; and a shore connecting section with a triangular cross-section. The $0.92 \mathrm{~m}$ shore connecting section is angled at $1: 15$ to the sloped beach.

For the Planar Laser induced Fluorescence (PLIF), the laser used was a Spectra-Physics 5W argon laser. A Lincoln laser scanner, was used to produce the laser sheet. The scanner and its housing were attached to a level gauge, which enabled it to be moved up and down a scale. Thus the height of the laser sheet can be adjusted, and for the horizontal laser sheet multi-layer images of dye plumes can be obtained. This is a novel way of "slicing" the depth of water into layers in order to study how the dye plume varies throughout the specified depth. The fluorescent dye used was Rhodamine-B. A filter was used to filter out the reflected laser light and retain the emission fluorescence. This filter was attached to the front of the camera lens to eliminate the reflected laser light and record the laser induced fluorescent light. To simulate outfall discharges the dye was discharged with a steel tube suspended at mid-depth of the water. This is a simplification as the initial dilution of the discharge is more complicated, and actual sewage outfall systems for instance, are designed differently. However due to the large scale of the flume this simplification is considered reasonable.

For capturing the images, a high performance monochrome CCD camera was used. The images are recorded and digitized as bitmap (*.BMP) images. The greyscale value of the images range from $0-256$, and the resolution of each grabbed image is $576 \times 768$ pixels. Once the images are obtained the quantitative analysis can be performed. This is done for the digitised images when dye discharge is stopped to study the dispersion or decay of the residual dye. Using MATLAB, the images are read into matrices of greyscale values and calibrated to neutralize absorption of the laser light by the released dye.

Calibration of the greyscale values of the images is necessary due to the non-uniform distribution of laser intensity and the absorption of laser energy by the fluorescent dye on the light pathways. For small-scale experiments with low dye concentrations the calibration is a simple procedure, since the absorption of the laser energy by the dye may be negligible. However for a large-scale experiment with high dye concentration, the absorption effect cannot be ignored. Unfortunately absorption greatly complicates the calibration procedure. The calibration procedure is detailed in Chen and Jirka (1999), and will not be presented here due to space constraints.

Following calibration, the total amount of dye in the flow can be calculated as time progresses by summing the remaining greyscale values of each image. The total number of pixels representing dye could also be obtained by inserting a counter in the processing program to count the the number of non-zero pixels. Maximum greyscale values may also be found by the processing program easily. This is repeated as necessary, and by analysing images on a large scale for each experiment, the concentration profile can be obtained. From the basic concentration profiles the secondary characteristics can be analysed. The test 
conditions are presented in Table 1. All experiments were carried out under subcritical, turbulent flow conditions.

The free stream velocity profiles were mainly measured with an Acoustic Doppler Velocimeter (ADV) with a three-dimensional down-looking probe. The visualisation was performed with discharge points placed around the perimeter of the headland (Figure 2). These discharge points are numbered R1 - R6, C, and L1 L6. The side of the shore-connecting section near the shoreline was avoided due to the sloped beach.

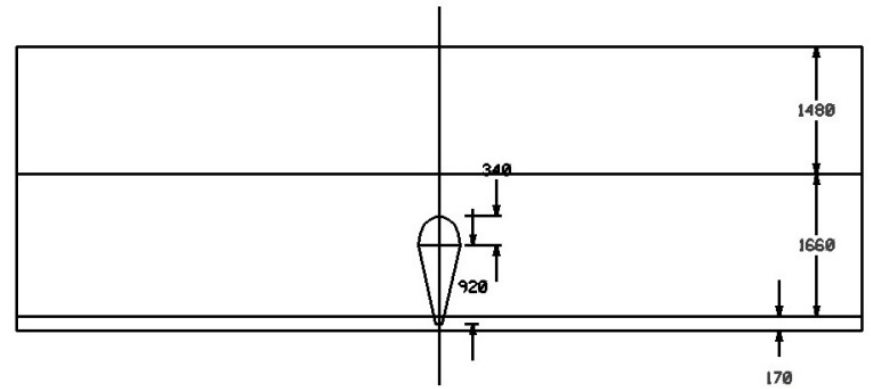

Figure 1: Schematic diagram of flume with sloped beach and hypothetical headland, all dimensions in mm

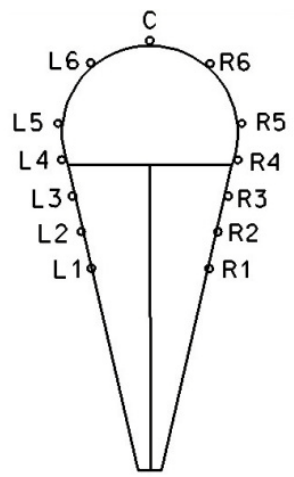

Figure 2: Discharge points around the perimeter of the headland model

Table 1: Initial flow parameters and heights of PLIF layers

\begin{tabular}{|l|l|l|l|l|}
\hline Layer & $\begin{array}{l}\text { Layer } \\
\text { height } \\
\text { from } \\
\text { bed } \\
(\mathrm{mm})\end{array}$ & $\begin{array}{l}\text { Depth } \\
\mathrm{H}\end{array}$ & $\begin{array}{l}\text { Averaged } \\
\text { free stream } \\
\text { velocity, U } \\
(\mathrm{m} / \mathrm{s})\end{array}$ & $\begin{array}{l}\text { Averaged } \\
\text { Reynolds } \\
\text { number, } \\
\mathrm{Re}_{\mathrm{h}}\end{array}$ \\
\hline 1 & 70 & 100 & 0.07 & 6223 \\
\hline 3 & 50 & 100 & 0.07 & 6223 \\
\hline 5 & 30 & 100 & 0.07 & 6223 \\
\hline
\end{tabular}

\section{Results}

\subsection{Flow patterns and plume formation with dye discharge}

In real life situations, if a large amount of pollutant is released in the waters before a stable plume forms to convey the pollutants to the free stream, this may impact on the water quality of the area. In the experiments, when dye is released at each discharge point, the time needed for a stable plume to be formed is recorded to study the plume formation characteristics as dye is discharged from different discharge 
Paper No.

points. The process is also observed and briefly described here. Figure 3 shows representative, fullydeveloped dye plumes at discharge points R1 and L1.

The observations presented here are based on dye discharge for Layer 1 (Table 1). For points R1 and L1, when the dye discharge started, accumulation of the released dye was seen at the discharge point before a dye plume was formed. As the amount of accumulated dye increased, movement towards the free stream was seen, where the flow velocity is comparatively high. One minute after the dye discharge started the accumulated dye has moved to the lee of the headland near the free stream, (in the region of points R5 and R6), and the released dye joined the free stream. A stable dye plume was established 30s later.

For points R2 and L2, the movement of the released dye to the free stream was quicker. The dye plume was stabilized one minute after starting discharge.

For points R3 and L3, movement to the free stream was quicker because these points were near the mixing region, in between the shore area and free stream. The accumulated dye started to disperse about 20s after discharge started, and 40s after discharge started a stable dye plume was established.

For points R4 and L4, and R5 and L5, as soon as the dye discharge was started the released dye moved towards the head in the same region near the lee. No accumulation of dye was seen. A stable dye plume was established 20s after dye discharge was started.

For points R6, C and L6, the dye plume was established as soon as the dye discharge was started, as these positions were located near immediately adjacent to the free stream.

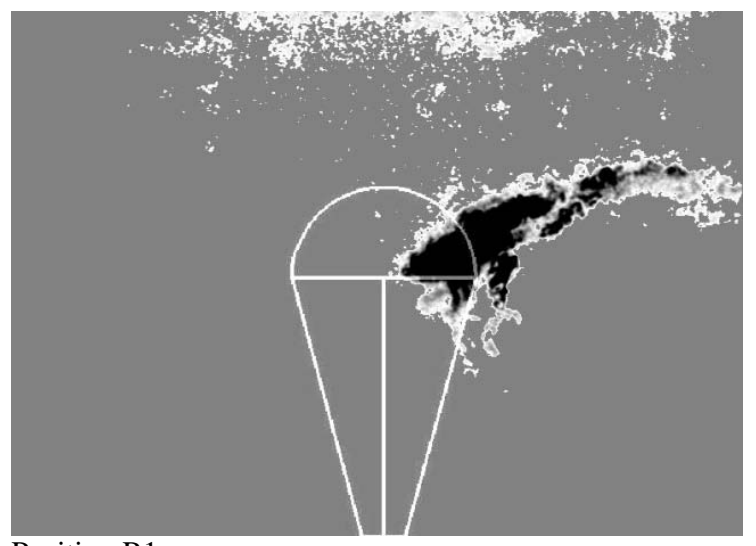

Position R1

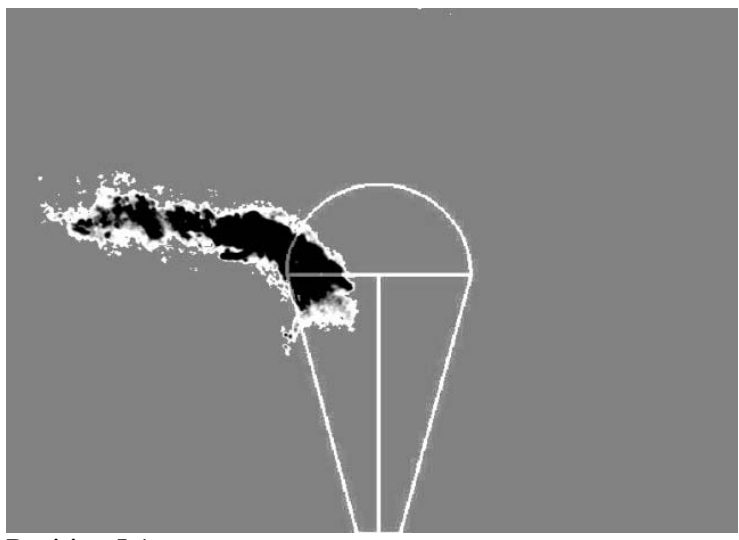

Position L1

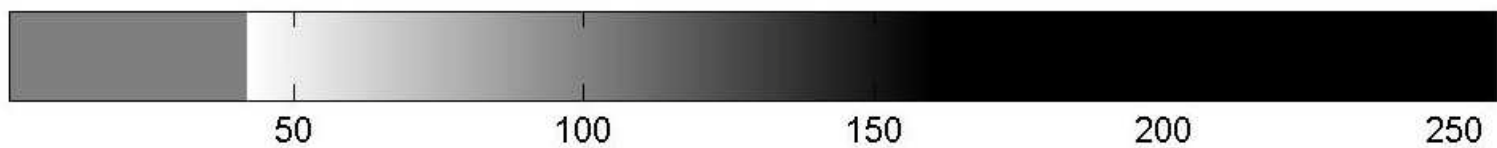

Figure 3: Planar laser-induced fluorescence (PLIF) images of dye concentration distribution for developed dye plumes for dye discharge at discharge points R. Colour bar indicates intensity of dye as greyscale.

\subsection{Flow patterns after dye discharge was stopped}

After an equal volume of dye was discharged for all cases, and the plume has been established, the dye discharge was stopped to record the time needed for flushing of the residual dye and study potential trapping behaviour. The observations presented here are based on dye discharge for Layer 1 (Table 1).

For points R1 and L1, at 2 minutes after the dye discharge was stopped, the residual dye visibly reduced significantly as shown for R1 in Figure 4. This was followed by about 8 minutes of visibly low concentration residual dye in the image. After a total of 10 minutes after dye discharge was stopped, most of the residual dye has been dispersed, but some traces of trapped dye were still visible after 30 minutes. This observation indicates a rapid initial dispersion of the trapped dye. Points R2 and L2 were very similar. For points R3 and L3, the residual dye was significantly reduced after 2 minutes. After a total of 10 minutes after dye discharge was stopped, a large portion of the residual dye has dispersed. There was little trapped residual dye visible. Therefore, trapping starts to decrease significantly with discharge at R3 and 
Paper No.

\section{L3. Points R4 and L4 were very similar.}

For points R5 and L5, the residual dye was quickly dispersed after 1 minute as shown for L5, and there was no trapped dye for these points. No trapping was also observed. Entrained residual dye was seen throughout the images after the discharge was stopped. It was observed that the concentration of the entrained dye increased after 4 or 5 minutes of stopping discharge. This is explained by the recirculatory flow. The recirculatory flow brings the residual dye away from the headland, and entrains it unto the recirculation zone, bringing masses of residual dye back towards the headland in a clockwise ellipse movement. The residual dye is then slowly dispersed by the main current. This indicates that the mixing region may serve as a mechanism to funnel entrained pollutants into the free stream where rapid dilution and mixing can occur.

For points R6, C and L6 the remaining dye quickly dispersed within 15 seconds, and 10 seconds for point C. There was no stagnant residual dye in any form. The recirculation behaviour was also not seen for dye discharge at points R6, L6 and C.
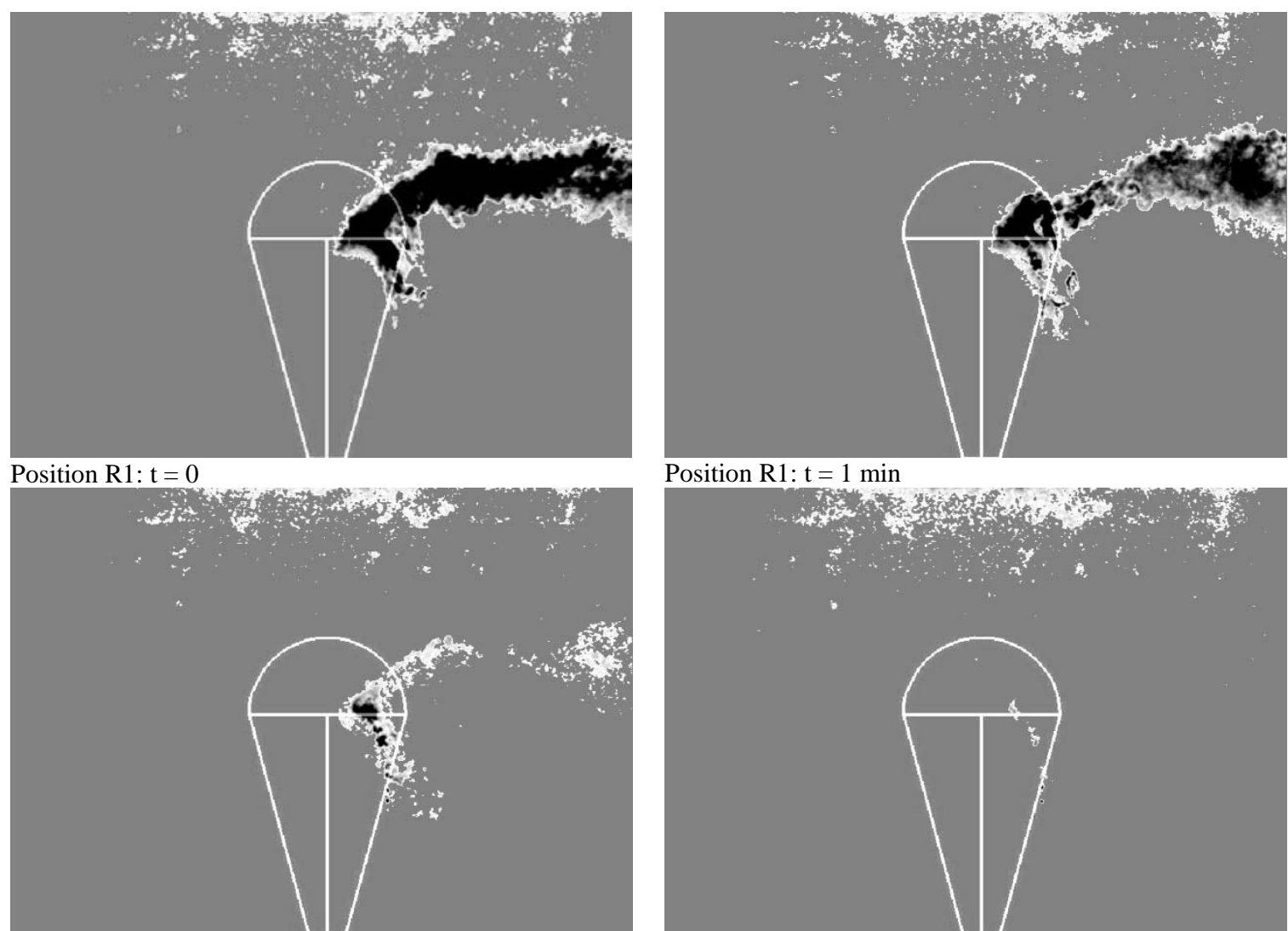

Position R1: $\mathrm{t}=2$ mins

Position R1: $\mathrm{t}=10$ mins

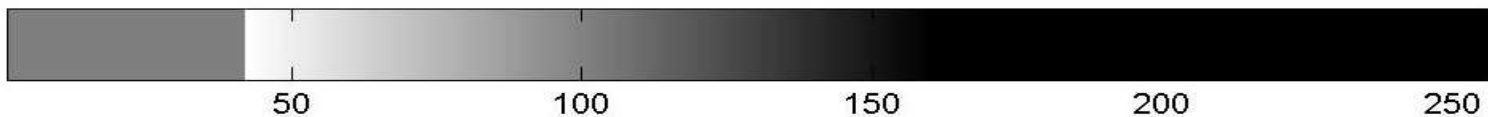

Figure 4: Planar laser-induced fluorescence (PLIF) images of dye concentration distribution after dye discharge was stopped at mirroring discharge points, Positions R1 and L12. Colour bar indicates intensity of dye as greyscale.

\subsection{Residual dye concentration decay profiles}

\subsubsection{General profile characteristics}

Total dye is defined as the sum of greyscale values of calibrated pixels that represent the dye in a single image. A total dye decay profile is the sum of greyscale values for each image plotted against time elapsed. The total dye decay plots are normalised with the highest total dye value in a single experimental series, and semilog plots of the normalised total dye are also presented. The total dye variation is a spatial 
variation. Maximum dye is defined as the highest single greyscale value $(0-255)$ in a single image. It is presented as a normalised profile, normalised with the highest value. Semilog plots of the normalised maximum dye are also presented. The maximum dye decay profile fluctuates significantly more than total dye. The dye pixel number is the sum of the counter inserted to count the number of pixels representing the dye, as opposed to pixels which represent the background of surrounding water.

\subsubsection{General dye decay profiles}

For layer 1, the dye decay profiles are mostly asymptotic(Figure 5), except for some discharge points located near the free stream. This is seen for total and normalised dye decay profiles. It was expected that plotting a semilog profile would result in a linear relationship, but this was not the case when the actual semilog profiles were produced.

Grouping behaviour is seen where dye decay profiles for R1 and R2 collapse into a similar curve while R3, R4 and R5 have different curves, and R6 and C also collapse into a similar curve together. This indicates that the residual dye decay behaviour may be influenced by different discharge locations, showing different flushing characteristics. Also, a slight second peak is seen in the decay profiles (normalised total dye) at R5, corresponding with the previous observed increased residual dye due to the recirculatory flow in section 3.2.

The maximum dye and dye pixel number profiles suggest that more residual dye is entrained in the recirculation zone for dye discharge nearer to the shore, rather than being channelled into the free stream. The normalised maximum dye profiles show the profiles clearly separated, with individualistic decay profiles. Decay profiles at discharge points near the shore are closer to the maximum normalised value while those further away from the shore and nearer to the free stream reach zero. Some grouping behaviour is evident with R1, R2, and R3 distinctly different from R4, R5, R6 and C. The semilog plot of the maximum dye profiles is also non-linear. The dye pixel number profile is also clearly separated for the discharge points, where the maximum dye pixel number for R6 and C is clearly lower. This shows that more residual dye is entrained in the recirculation zone for dye discharge nearer to the shore.

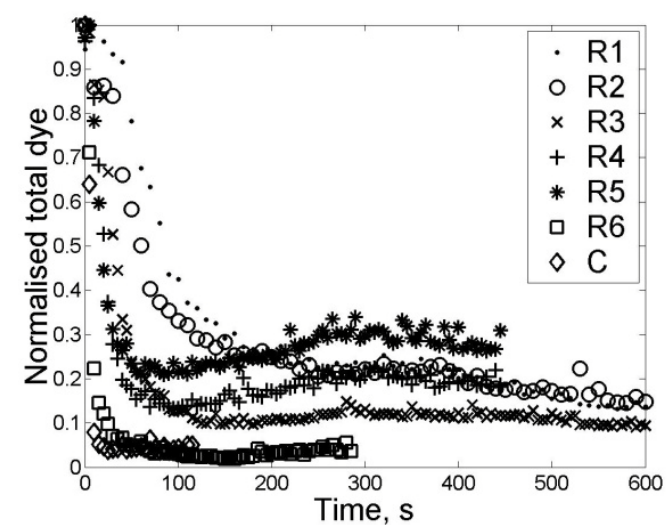

Normalised Total Dye Decay Profiles

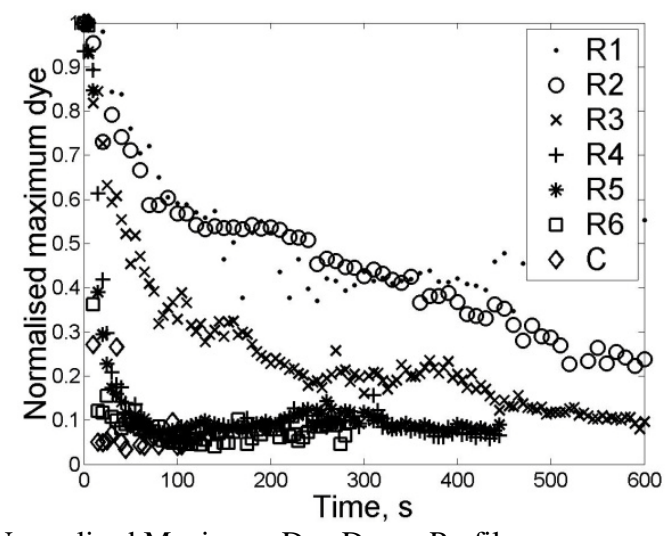

Normalised Maximum Dye Decay Profiles

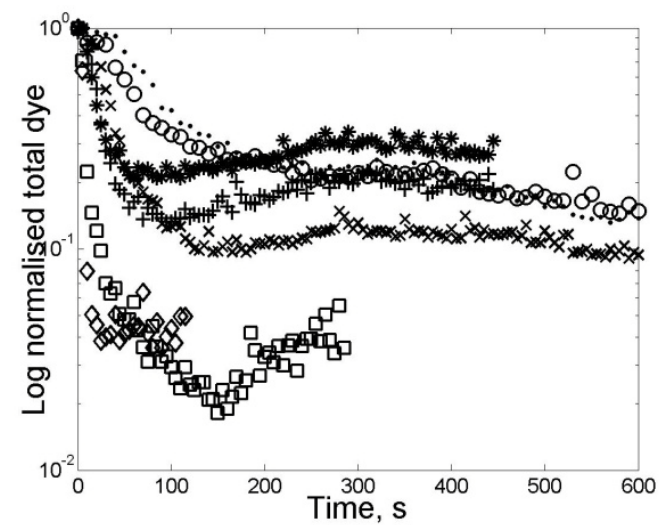

Semilog plot of normalised total dye decay profiles

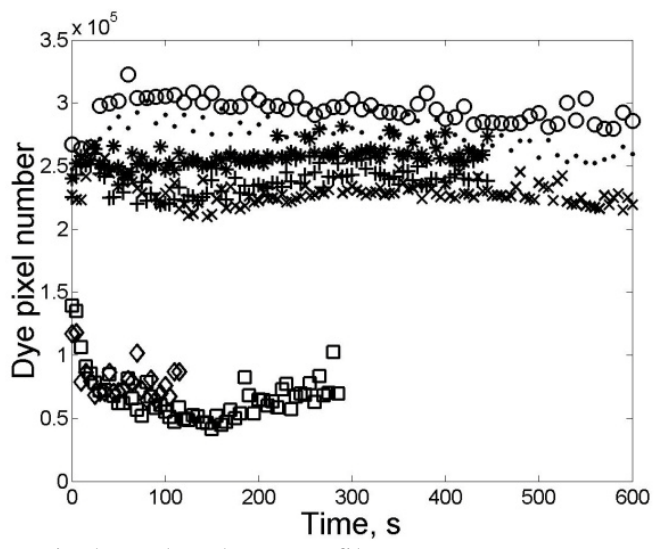

Dye pixel number decay profiles 
Coastal Dynamics 2009

Paper No.

Figure 5: Dye decay profiles at layer 1 for flushing of residual dye after dye discharge was stopped in steady flow, with absorption analysis incorporated. The same symbols are used for all plots, for plots without a legend please refer to any legend shown. Units of total and maximum dye are in milligram (mg) multiplied by a factor of $9.185 \times 10^{-8}$ for the laser sheet thickness of $2 \mathrm{~mm}$.

For layer 3, from the total dye decay profiles, a clear separation occurs with individualistic profiles for the discharge points, graduating from the shore to the free stream (Figure 6). The asymptotic dye profiles neatly lined up according to distance from the shore. Again near-shore points appear to take the longest time to decay. As for maximum decay profiles, the profiles fluctuated significantly as expected. Grouping seemed to occur as before, where R1, R2 and R3 formed one group with a similar profile, and R4, R5, R6 and $\mathrm{C}$ formed another group (see maximum dye decay profiles).

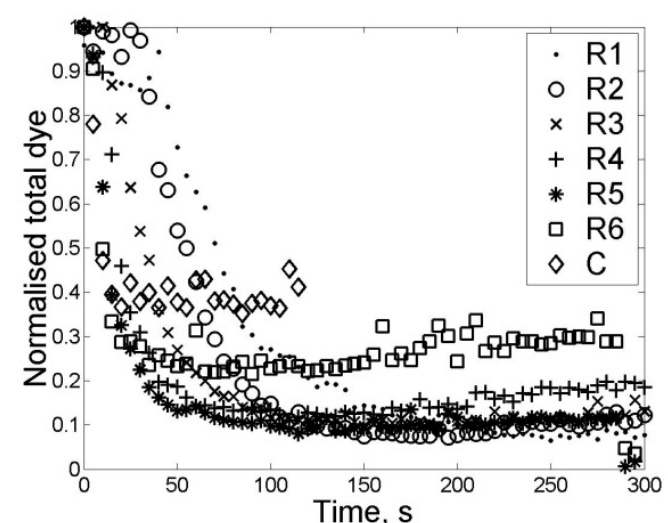

Normalised Total Dye Decay Profiles

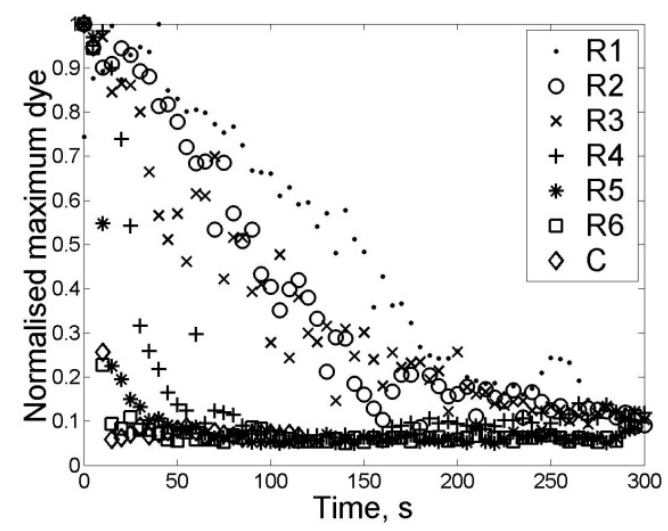

Normalised Maximum Dye Decay Profiles

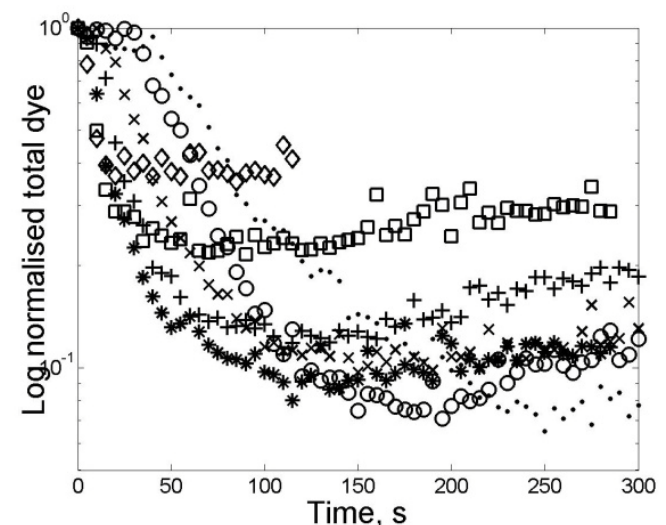

Semilog plot of normalised total dye decay profiles

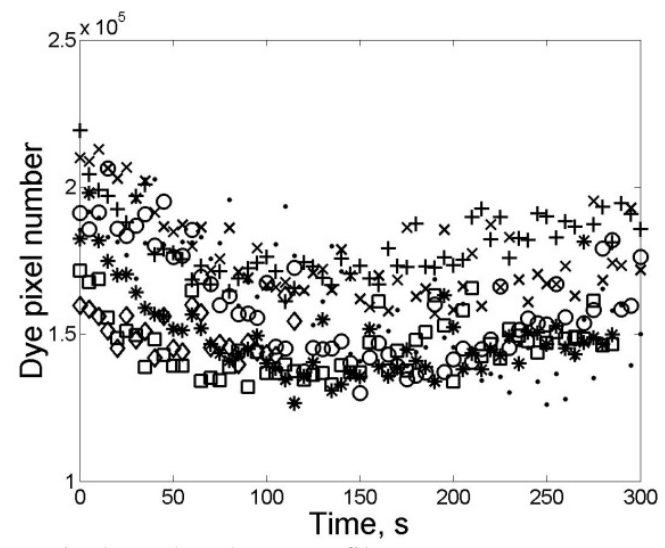

Dye pixel number decay profiles

Figure 6: Dye decay profiles at layer 3 for flushing of residual dye after dye discharge was stopped in steady flow, with absorption analysis incorporated. The same symbols are used for all plots, for plots without a legend please refer to any legend shown. Units of total and maximum dye are in milligram (mg) multiplied by a factor of $9.185 \times 10^{-8}$ for the laser sheet thickness of $2 \mathrm{~mm}$.

For layer 5, the dye profiles are quite similar to layer 3 (Figure 7). The dye decay profiles were again separated according to discharge position. Grouping behaviour is also seen but less clear. No grouping was seen for the maximum decay profiles, although separation of the decay profiles was seen. 


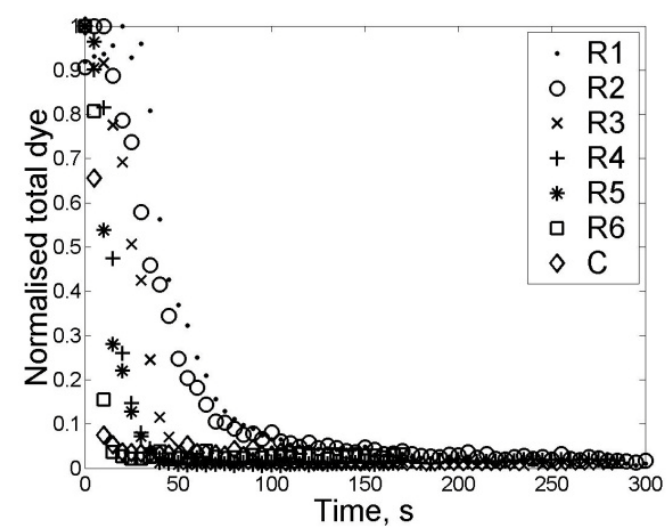

Normalised Total Dye Decay Profiles

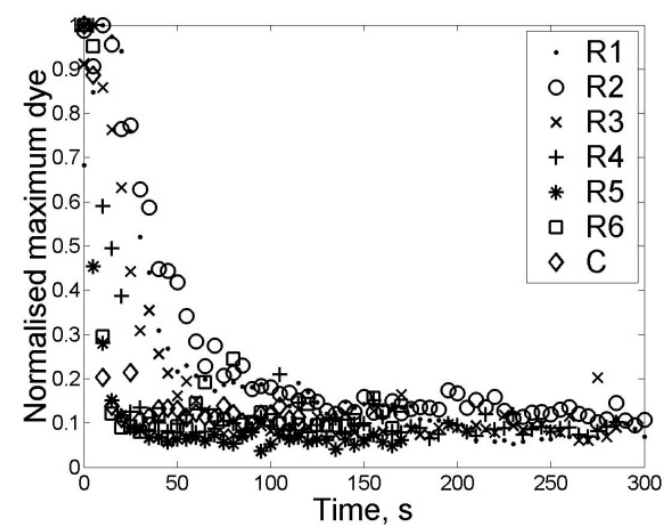

Normalised Maximum Dye Decay Profiles

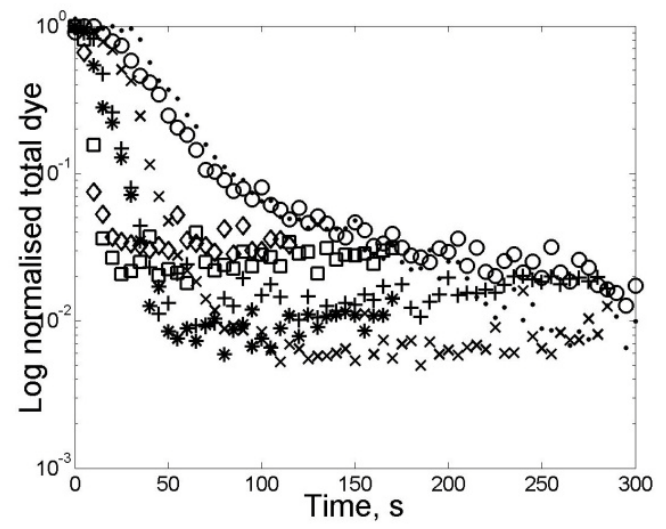

Semilog plot of normalised total dye decay profiles

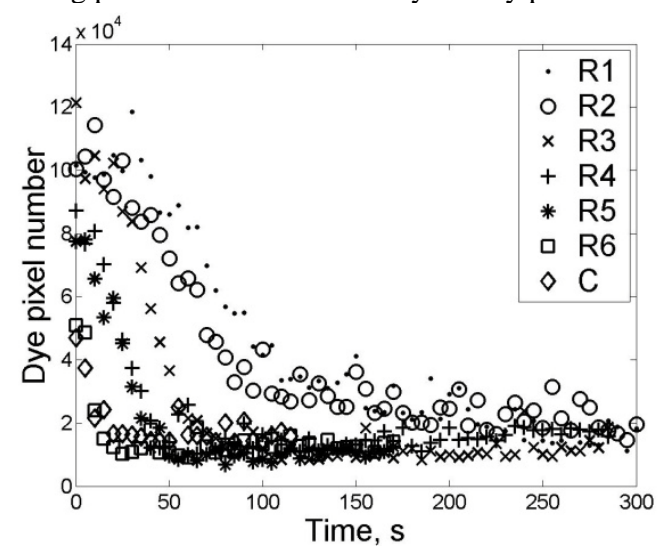

Dye pixel number decay profiles

Figure 7: Dye decay profiles at layer 5 for flushing of residual dye after dye discharge was stopped in steady flow, with absorption analysis incorporated. The same symbols are used for all plots, for plots without a legend please refer to any legend shown. Units of total and maximum dye are in milligram (mg) multiplied by a factor of $9.185 \times 10^{-8}$ for the laser sheet thickness of $2 \mathrm{~mm}$.

\subsubsection{Secondary dye decay profile characteristics}

In order to quantify dye decay between discharge positions, gradients (slopes) of the dye decay profiles were calculated. This has been presented in Chen, et al. (2003). The gradient is calculated based on the initial stage of decay (please refer to Stage 1 in (Chen, et al. 2003)) and incorporated into the processing programs. The semi-logarithmic profile is linearly-regressed only at Stage 1 and gradients calculated.

To characterise the dye decay against distance of dye discharge from the shore, the obtained gradients were plotted against distance of discharge position from the shore, normalised against the width of the beach. The gradients mostly ranged in value from 0 to about 0.1 (Figure 8 ). The trend showed that the decay gradient increased farther away from the shore. This is expected, as the high free stream velocity ensures a faster flushing time. This is also consistent with the experimental observations. However, the residual dye would remain entrained in the recirculation zone for a significantly longer time for discharge points nearer to the shore. This is consistent with the previous experimental observations, and supports the classification of flow behaviour according to location. 


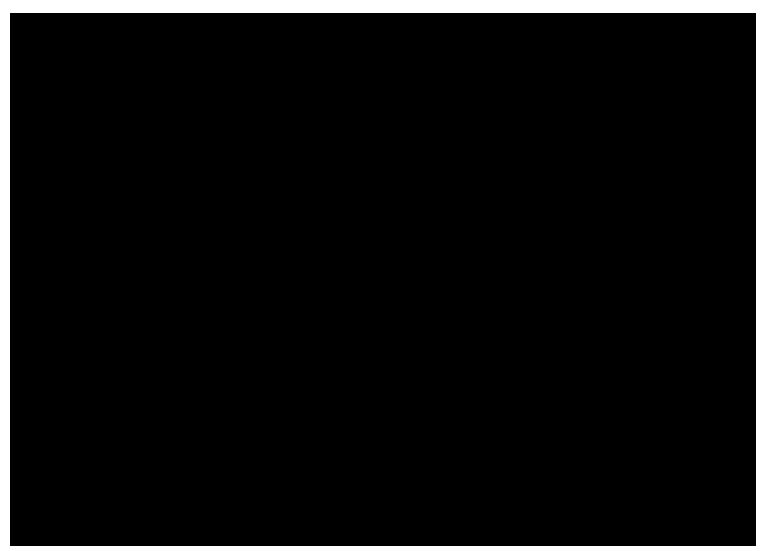

Figure 8: Gradients of dye decay profiles at layers 1, 3 and 5 for flushing of residual dye after dye discharge was stopped in steady flow

In addition to the decay gradients, the stationary point where the derivative of the dye decay profile is null $(\mathrm{dy} / \mathrm{dx}=0)$ is also presented here (Figure 10). This stationary point is named the "low concentration threshold point” in this study. The low concentration threshold point indicates when the residual dye decay profile becomes asymptotic. In real life situations this will indicate when the concentration of the residual pollutants decreases to a very low level.

Figure 9 shows an example of the calculated low concentration threshold point for the total dye decay profile at layer 1 for discharge at R3. The derivative of a decay profile is taken throughout the entire profile along regular intervals, and the derivatives that are close to null are extracted. This array is then matched with the time vector. The first time vector value for a null derivative is defined as the low concentration threshold point.

To characterise residual dye decay against distance of discharge from the shore, the obtained low concentration threshold points for each residual decay profile were plotted against normalised distance from the shore, and again the general trend was the dye decayed faster farther away from the shore (Figure 10). In addition, the low concentration threshold point was mostly below 100 s.

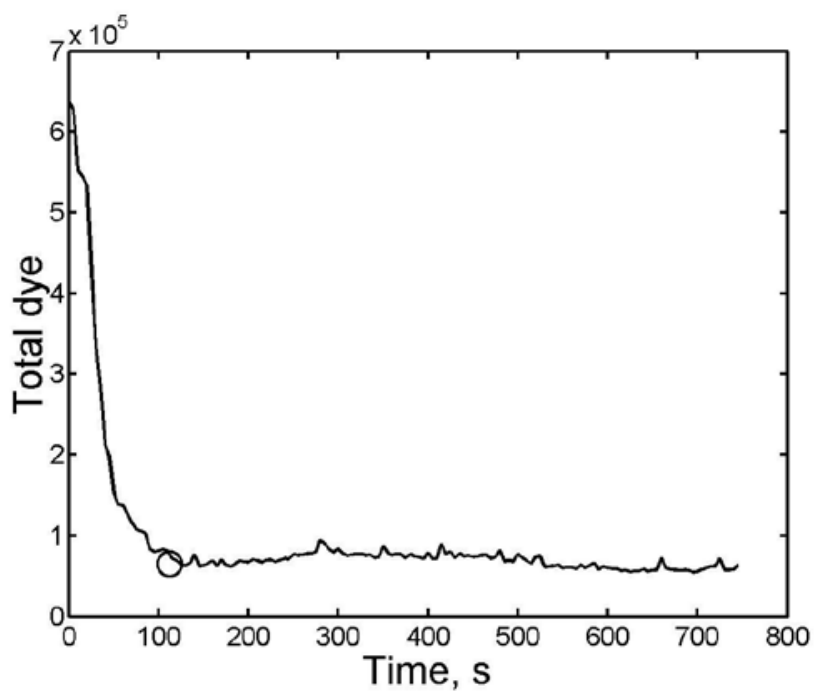

Figure 9: Example of calculated low concentration threshold point for total dye decay profile at layer 1, dye discharge at point R3. Circle denotes low concentration threshold point. 
Coastal Dynamics 2009

Paper No.

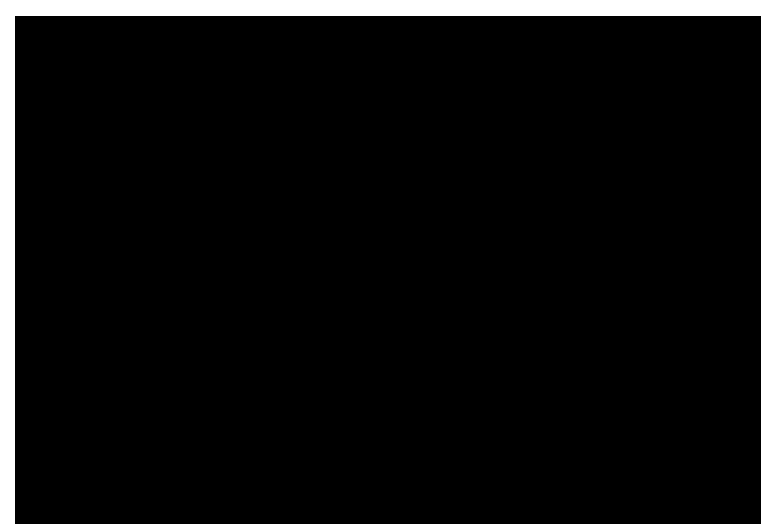

Figure 10: Low concentration threshold points of dye decay profiles at layers 1, 3 and 5 for flushing of residual dye after dye discharge was stopped in steady flow

\section{Discussion}

In real life situations siting of pollutant outfalls such as wastewater outfalls is a crucial consideration in the construction and economic aspects of such coastal projects. Additional expenditure will be incurred if the outfall needs to be located far out into the main current of the ocean to ensure the water quality of the coastal near shore areas. Intensive engineering works and analysis of pollutant mixing and transport will be necessary. While the water quality of the region is of the utmost importance, economics is an important factor towards the implementation of such coastal projects. Thus a compromise needs to be achieved between environmental considerations and costs.

These experiments are performed for a hypothetical headland located on a sloped beach to represent real coastal situations. In this study, the effects of varying discharge points, and the recirculatory flow mechanism is explored, and the results indicate flow behaviour and pollutant dispersion characteristics in such a coastal situation. For real life projects, further modeling work may be necessary to account for more geographical features and the bathymetry of the coastline.

In order to make the experiment possible, distorted scales are used for the water depth and headland. In real life situations, the water depth in such areas would be in the order of $10 \mathrm{~m}$. However, the headland could be in the other of up to $10^{2}$ or $10^{3} \mathrm{~m}$. High experimental flow velocities ensure sufficiently high Reynolds numbers for the ambient flow. Further studies with tidal (oscillating) flow, and pollutant dispersion within the headland-generated recirculation zone will be presented in future. The steady flow condition explored in this study presents a "worst-case scenario" where there is no variation in the flow velocity and direction. This is to create the most favourable conditions for pollutant trapping in the experimental set-up.

From the results, it seems that for siting of pollutant outfalls the ideal points should not be nearer to the shore than points L6, C and R6 for the headland perimeter based on the current experimental set-up. At these locations the discharged dye was dispersed quickly and no residual dye was entrained into the recirculation flow. However, in real life situations, it may not be possible to site the outfalls so far out into the ocean due to physical or cost constraints. From the experimental results it is suggested that the best alternative location would be points R5 and L5 for pollutant discharge at the perimeter of the headland. It has been proven that for discharge at this location, little dye is entrained into the recirculatory flow and no trapping occurs. Although it is subject to the unstable velocity fluctuations of the mixing region, the released dye is still dispersed efficiently.

It is expected that similar results will be obtained if the experiment is repeated. Although the flow conditions of the experiments can be repeated, it is impossible to duplicate the exact flows under which the results are obtained. Thus a small discrepancy is expected for both sides of the headland, and also between layers. It is reasonable to assume that major large-scale characteristics, such as presence and position of trapping will be consistent, while other minor characteristics such as shape of dye plume may differ slightly. 
The experiment was repeated with high spatial variability settings as a precaution for any unexpected flow behaviour. The flow parameters were the same, while the overhead camera lens was changed to a $8.5 \mathrm{~mm}$ lens. The experiment was only repeated for layer 1, as shown in Figure 11 the high spatial variability results were very similar to the low spatial variability results. The near-shore discharge points, R1 and R2 collapsed together for the total dye decay profile, mixing zone discharge points R3 - R6 also grouped together. Point $\mathrm{C}$ was more erratic. Grouping was also seen for the maximum dye decay and total number of dye pixel profiles.

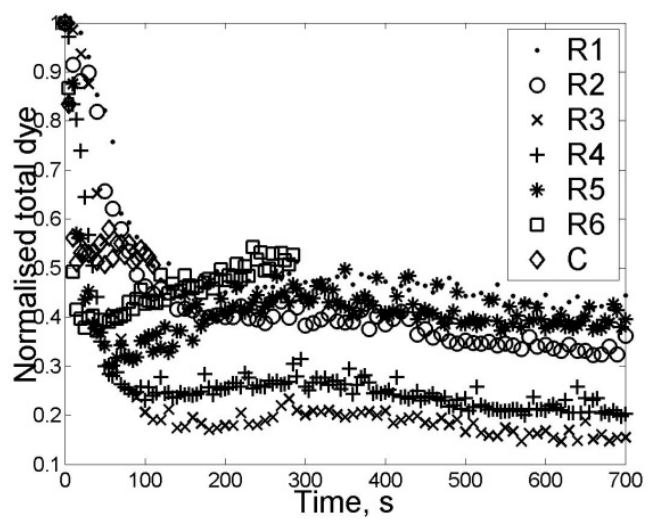

Normalised Total Dye Decay Profiles

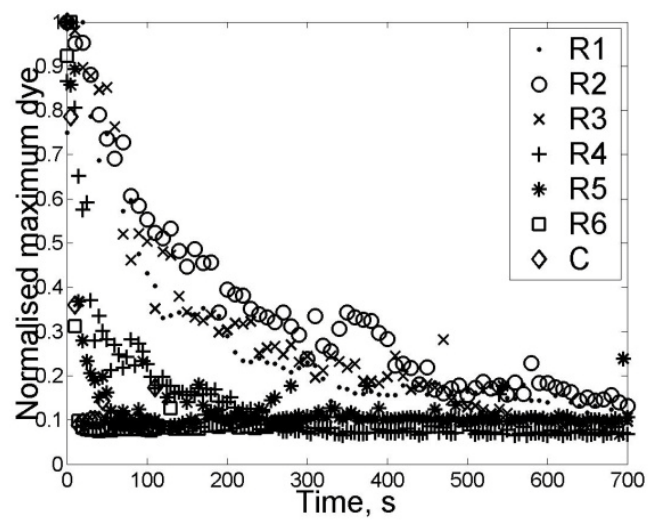

Normalised Maximum Dye Decay Profiles

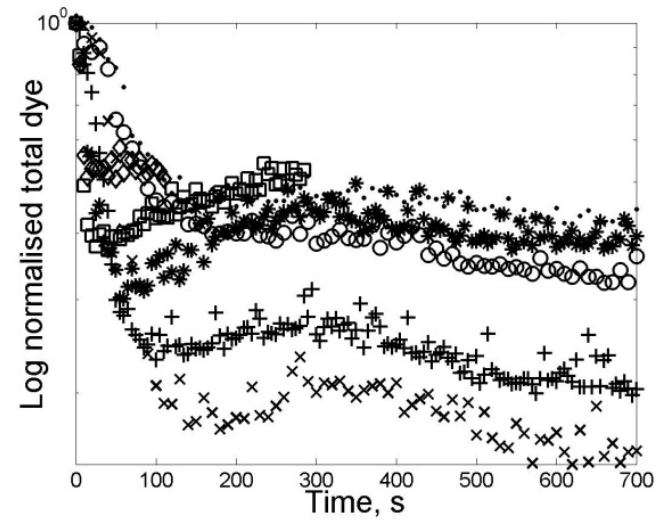

Semilog plot of normalised total dye decay profiles

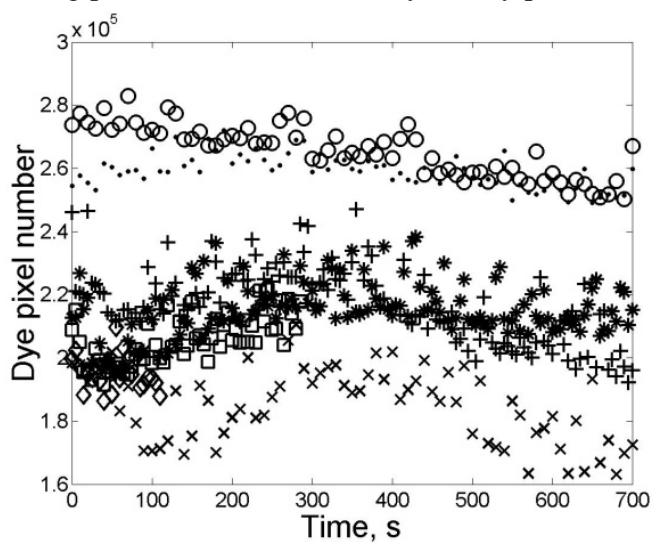

Dye pixel number decay profiles

Figure 11: Dye decay profiles at layer 1 for flushing of residual dye after dye discharge was stopped in steady flow, with absorption analysis incorporated, high spatial variability. The same symbols are used for all plots, for plots without a legend please refer to any legend shown. Units of total and maximum dye are in milligram (mg) multiplied by a factor of $2.25 \times 10^{-7}$ for the laser sheet thickness of $2 \mathrm{~mm}$.

\section{Conclusion}

It is seen that dye decay profiles as presented in this study is useful to indicate flushing characteristics. For real life situations, scaling and modeling of existing coastlines and geographical features can be performed to obtain flushing characteristics during the design stage of the project. As for the secondary analysis, the low concentration threshold method seems to be effective at predicting the general trend. From the experiments, flow behaviour was also separated according to discharge location, where the residual dye decays at a rate according to its position away from the shore. This leads to some grouping behaviour where discharge points nearer to the shore have a different decay profile from discharge points nearer to the free stream, or open waters in real life situations. The experiments have proved that such coastal areas can be divided and analysed as the free stream, mixing region and near shore areas. Although dye discharged near the shore will result in significantly longer flushing times, it seems that the mixing region may be effective in dispersing pollutants although flow behaviour is more complicated at this region. As a 
precaution, the mixing region should be treated as a potential trapping zone similar to the near shore area, although the degree to which trapping occurs is significantly diminished.

\section{Acknowledgements}

Professor Daoyi Chen's supervision and guidance for this study is gratefully acknowledged. This study is supported by a grant (GR/L/34570) from the UK Engineering and Physical Science Research Council (EPSRC). F.E. Tang's PhD study was also partially supported by an UK Overseas Research Studentship.

\section{References}

Babarutsi, S., Chu, V.H. 1991. Dye-concentration distribution in shallow recirculating flows, Journal of Hydraulic Engineering, 117(5)

Babarutsi, S., Ganoulis, J., and Chu, V.H. 1989. Experimental investigation of shallow recirculating flows, Journal of Hydraulic Engineering, 115(7)

Chen, D., and Jirka, G.H., 1991. Pollutant mixing in wake flows behind islands in shallow water, in Proc. of the Int. Symp. on Environmental Hydraulics, Hong Kong, 1991

Chen, D., and Jirka, G. H., 1995. Experimental study of plane turbulent wakes in a shallow water layer, Fluid Dynamics Research, 16

Chen, D., and Jirka, G. H., 1997. Absolute and convective instabilities of plane turbulent wakes in shallowwater layer, Journal of Fluid Mechanics, 338

Chen, D., and Jirka, G.H., 1999. LIF study of plane jet bounded in shallow water layer, Journal of Hydraulic Engineering, 125(8)

Chen, D., Tang, F.E., Chen, C., 2003. PLIF study of pollutant trapping and 3-D plume structures in recirculation zones around a headland: steady and tidal currents, In Proc. of the Int. Symp. on Shallow Flows, ASCE, Holland, 2003

Chen, D., Tang, F.E., and Chen, C., 2005. Pollutant trapping at a coastal headland, Journal of Waterway, Port, Coastal and Ocean Engineering, 131(3)

Chen, et al., 2007. Boundary Layer Structure of Oscillatory Open-Channel Shallow Flows over Smooth and Rough Beds, Experiments in Fluids, 42(5)

Ingram, R. G., and Chu, V. H., 1987. Flow around islands in Rupert Bay: An investigation of the bottom friction effect, Journal of Geophysical Research, 92(C13)

Li, M., et al., 2007. Development of a quasi-3d numerical model for sediment transport in the coastal region, Journal of Hydro-Environment Research, 1(2)

Lloyd, P.M., Stansby, P.K., 1997. Shallow-water flow around model conical islands of small side slope. I: surface piercing, Journal of Hydraulic Engineering, 123(12)

Lloyd, P.M., Stansby, P.K., Chen, D., 2001. Wake formation around islands in oscillatory laminar shallowwater flows, Journal of Fluid Mechanics, 429

MacDonald, D.G., and Jirka, G.H., 1997. Characteristics of headland wakes in shallow flow, In Proc. of the Congress of the IAHR, IAHR , Netherlands, 1997

Wolanski, E., Imberger, J., Heron, M.L., 1984. Island wakes in shallow coastal waters, Journal of Geophysical Research, 89(C6) 